\title{
Correlação entre a densidade óssea mandibular, femural, lombar e cervical
}

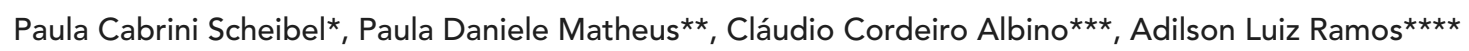

\section{Resumo}

Introdução: diante da maior frequência de pacientes adultos que se submetem atualmente ao tratamento ortodôntico, as condições gerais de saúde dessa faixa etária têm sido motivo de investigações correlacionadas aos eventos ligados ao metabolismo ósseo, haja vista que os movimentos dentários são dependentes do processo de remodelação óssea, ainda que num nível local. Diferentes padrões de densidade óssea podem acarretar diferentes respostas ao movimento ortodôntico. Objetivos: o presente estudo avaliou a correlação da densidade mineral óssea (DMO) geral com aquela da região mandibular. Métodos: para tanto, 22 mulheres saudáveis, com idades entre 30 e 45 anos, foram selecionadas para os exames de densitometria óssea das regiões lombar, cervical e femural, bem como da região mandibular. Foram testadas as correlações entre essas leituras e, também, estabelecidos valores de referência para as áreas cervical e mandibular. Resultados: os resultados não demonstraram correlação significativa entre a densidade mandibular e as demais áreas estudadas. Houve correlação significativa apenas entre a região cervical e a femural. O valor médio DMO normal para a região mandibular foi de $0,983 \mathrm{~g} / \mathrm{cm}^{2}$ (d.p. $=0,334)$, enquanto para a região cervical foi de $0,768 \mathrm{~g} / \mathrm{cm}^{2}$ (d.p. $\left.=0,102\right)$, e os valores médios para a região lombar e femural foram de, respectivamente, $1,127 \mathrm{~g} / \mathrm{cm}^{2}$ (d.p. $=0,067$ ) e $0,925 \mathrm{~g} / \mathrm{cm}^{2}$ (d.p. $=0,078$ ), esses últimos semelhantes aos valores de referência da Organização Mundial de Saúde. Conclusões: sugere-se que o exame da área femural possa abranger o valor esperado para a área cervical, entretanto há necessidade do exame densitométrico particular para a área mandibular, não sendo adequada a extrapolação dos valores tradicionais (lombar e femural) para estimativa dessa área. Estudos adicionais são necessários para avaliar as variações densitométricas locais e eventual influência sobre a movimentação ortodôntica.

Palavras-chave: Densitometria óssea. Osteoporose. Movimentação ortodôntica. Remodelação óssea. Densidade óssea mandibular.

\footnotetext{
* Graduada em Odontologia pela Universidade Estadual de Maringá (UEM). Especializanda em Ortodontia pelo CESUMAR/Dental Press. Mestranda em Odontologia Integrada pela UEM.

** Graduada em Odontologia pela UEM.

*** Graduado em Medicina pela Universidade Federal do Paraná (UFPR). Especialista em Endocrinologia e Metabologia pelo Hospital de Clínicas da UFPR. Mestre em Medicina Interna pela UEL. Doutor em Ciências Médicas - Radiologia pela Universidade de São Paulo (USP/ABC).

**** Professor adjunto do Departamento de Odontologia da UEM. Mestre em Ortodontia pela Faculdade de Odontologia de Bauru (USP/BAURU). Doutor em Ortodontia pela Universidade Estadual Paulista Júlio Mesquita Filho (Unesp/ Araraquara). Professor dos cursos de especialização em Ortodontia da ABO, CESUMAR/Dental Press e UEM.
} 


\section{INTRODUÇÃO}

A osteoporose é uma patologia caracterizada pela diminuição de massa óssea e pela deterioração estrutural do tecido ósseo, causando fragilidade óssea e suscetibilidade acentuada a fraturas, especialmente da coluna, quadril e punho, embora qualquer osso possa ser afetado ${ }^{20}$. O método mundialmente empregado para o diagnóstico dessa alteração é o uso do densitômetro duo-energético por raios X, também denominado DXA (Dual Energy X-Ray Absorptiometry), o qual pode ser empregado para avaliações de densidades ósseas em qualquer área do esqueleto ${ }^{25}$.

A absorciometria duo-energética por raios $\mathrm{X}$ (DXA) foi introduzida em 1987 pela empresa Hologic Inc. (EUA), substituindo os radioisótopos por tubos de raios $\mathrm{X}$ como fonte de radiação. $\mathrm{Na}$ DXA, o intenso fluxo de fótons no interior do tubo de raios X permite que haja uma redução de até 6 minutos no processo de escaneamento da área de interesse no esqueleto ósseo. A resolução e a precisão do equipamento foram sensivelmente melhoradas, uma vez que a DXA manteve compatibilidade com as dados utilizados pela absorciometria duofotônica (DPA), sistema precursor de medição de massa óssea. $\mathrm{Na}$ absorciometria, a fonte energética de fótons pode utilizar tanto radioisótopos como tubos de raios $\mathrm{X}$, a qual se encontra no interior de uma ampola. Os fótons atravessam os tecidos moles e ósseos do paciente, ascendendo e penetrando em um detector, onde a intensidade do feixe é registrada. O princípio operacional da absorciometria duo-energética está baseado nas características de atenuação diferenciais entre tecido ósseo e tecido mole pela passagem do feixe de radiação $\mathrm{X}^{28}$.

Embora a coluna lombar e o fêmur já apresentem um padrão de massa óssea definido e referendado pela Organização Mundial de Saúde (OMS) - o que permite um diagnóstico conclusivo com relação à osteoporose -, um local adequado para a determinação da densidade óssea no esqueleto craniofacial ainda não foi precisamente definido ${ }^{6,17}$.

$\mathrm{Na}$ Odontologia, considera-se a necessidade de reflexão sobre a morfologia dos ossos faciais e a definição de valores de densidade óssea, haja vista o envolvimento da qualidade óssea em áreas como Implantodontia, Ortodontia e Periodontia ${ }^{6,29}$.

Em 1995, Scarparo ${ }^{23}$ propôs um método para avaliar a densidade óptica óssea mediante a utilização de penetrômetro de alumínio. Nesse mesmo ano, Garcia ${ }^{5}$ avaliou a densidade óptica alveolar na região entre incisivos laterais e caninos, com um método radiográfico semelhante. Entretanto, em 1996, Kannis ${ }^{13}$ observou que uma radiografia convencional só permite a visualização da alteração óssea quando ocorre a diminuição de massa óssea numa escala de 30 a 50\%. E Horner et al. ${ }^{11}$ sugeriram que somente seria possível o diagnóstico de osteoporose por radiografias dentárias se o padrão ósseo dentoalveolar apresentasse correlação com outros sítios esqueléticos.

Polido $^{22}$ utilizou a metodologia radiográfica digital direta para avaliar alterações ósseas ao redor de implantes dentários durante o período de osseointegração. Concluiu que o sistema empregado no seu estudo foi eficiente. Porém, sugeriu que se criassem mais parâmetros de comparação, para consolidar a avaliação pelo sistema digital.

A comunidade científica vem, há muito tempo, preocupando-se em correlacionar as alterações morfofisiológicas dos ossos maxilomandibulares com alterações ósseas sistêmicas e aos fatores externos de componentes inflamatórios - como a doença periodontal -, esclarecendo os processos de reabsorção óssea alveolar transversal e horizontal nas cristas alveolares ${ }^{2,6,7}$. Entretanto, ainda há dúvida quanto à correlação osteometabólica e do padrão morfofisiológico para os ossos de suporte dentário, bem como para os demais ossos do esqueleto humano $6,8,16,21,24,26,29$.

O número de pacientes adultos nos consultórios de Ortodontia tem aumentado. Diante disso, aumentou também a preocupação dos ortodontistas e pesquisadores com relação às prováveis diferenças biológicas em resposta à movimentação ortodôntica. Estudos com populações hipertensas, 
diabéticas ou com osteoporose, entre outras alterações sistêmicas mais comuns nos adultos, têm sido realizados.

Consolaro $^{3}$ relatou que alguns fatores podem influenciar na resposta biológica diante da movimentação ortodôntica. Entre eles, o formato das raízes dentárias e o formato da crista alveolar. Esses fatores estariam relacionados com o grau de concentração de forças nas regiões apicais radiculares. Raízes mais triangulares e cristas alveolares mais retangulares acarretariam maior concentração de força no ápice radicular, favorecendo o processo de reabsorção do cemento.

Horiuchu, Hotokezaka e Kobayashi ${ }^{10}$ observaram que a movimentação dentária contra uma área de maior densidade óssea, por exemplo a cortical óssea, relaciona-se à maior reabsorção dentária.

Portanto, a osteoporose - ou simplesmente os diferentes níveis de densidade óssea, por estarem vinculados diretamente ao metabolismo ósseo - é particularmente instigante à comunidade ortodôntica, haja vista que uma perda mineral poderia influenciar o processo de reparação óssea alveolar; enquanto níveis de densidade maiores poderiam assemelhar-se ao efeito de corticais mais espessas, acarretando menores deflexões, maiores concentrações de forças nas raízes e, por consequência, maior probabilidade de reabsorções radiculares durante a movimentação ortodôntica. Entretanto, a literatura carece de publicações do gênero. Esse fato motivou o desenvolvimento de uma linha de pesquisa que inclui o presente trabalho.

Especificamente, o presente estudo teve como objetivo estabelecer padrões de normalidade da densidade óssea mandibular e da vértebra C3, e avaliar a correlação desses com os padrões densitométricos preestabelecidos da região Ll-L4 e femural.

\section{MATERIAL E MÉTODOS}

A amostra do presente trabalho foi composta por 22 mulheres saudáveis e não-menopáusicas, com idades entre 30 e 45 anos. Um consentimento esclarecido e assinado foi a condição primária para a inclusão de cada participante. A presença da maioria dos dentes nos arcos dentários, ausência de tratamento ortodôntico prévio e ausência de histórico de osteoporose e hiperparatiroidismo foram os demais critérios de inclusão.

O presente estudo foi devidamente aprovado pelo Comitê Permanente de Ética em Pesquisa Envolvendo Seres Humanos (COPEP) da Universidade Estadual de Maringá, sob o parecer $n^{\circ} 139 / 2006$.

As pacientes foram submetidas ao exame de densitometria óssea - realizado por um densitômetro DXA (Fig. 1) da marca Lunar, modelo Prodigy 8743 (GE Medical Systems), fabricado em junho de 2003, com kilovoltagem de $76 \mathrm{kV}$, amperagem de $3,00 \mathrm{~mA}$, dose de $3,70 \mathrm{mren}$ e com exposição que pode variar de 43 segundos a 1 minuto, dependendo do peso e altura da paciente.

A partir desse exame, foram obtidas as densidades ósseas do fêmur total direito, da região lombar (vértebras L1-L4), da terceira vértebra cervical (C3) e da região mandibular, sendo que os exames para área femural e lombar seguiram um protocolo padrão reconhecido internacionalmente e os resultados dessas regiões serviram de leitura controle.

Antes que as densitometrias ósseas fossem iniciadas, as voluntárias foram pesadas e tiveram suas alturas medidas para preenchimento desses dados no software do densitômetro.

Para realização da densitometria femural, a paciente era posicionada em decúbito dorsal sobre a mesa do equipamento, com os joelhos e pernas virados para dentro e os pés seguros com faixas em um suporte posicionador de forma triangular (Fig. 2). Um filete de luz laser (vermelha) incidindo sob a região central da coxa direita, e imediatamente abaixo da sínfise púbica, servia de referência para o posicionamento (Fig. 2).

Em seguida, com a paciente na mesma posição, foi colocado um bloco de espuma embaixo de suas pernas para que a densitometria da 
região lombar pudesse ser executada (Fig. 3). Nesse exame, o aparelho iniciou seu funcionamento em uma posição correspondente à quinta vértebra lombar. A luz laser era direcionada em um ponto $5 \mathrm{~cm}$ abaixo do umbigo, e o aparelho escaneava a paciente até a vértebra T12 (Fig. 3).

Os exames para as áreas C3 e mandibular foram obtidos com a voluntária em posição de decúbito ventral, com a face esquerda encostada sobre a mesa do equipamento, de modo a expor paralelamente a mandíbula desse lado ao feixe radiográfico - dessa forma, simulando uma tomada radiográfica facial a $45^{\circ}$ (oblíqua da mandíbula). Para que o exame fosse iniciado, o filete de luz laser era posicionado $3 \mathrm{~cm}$ acima da orelha da paciente, sendo finalizado logo abaixo da região correspondente à vértebra $\mathrm{C} 3$.

A figura 4 ilustra as regiões de interesse (Region of Interest - ROI) para avaliação da densidade óssea da região femural, lombar (segmento L1-L4), mandibular e cervical (C3).

Os dados obtidos foram comparados empregando-se um programa de estatística (Statistica, New York, EUA).

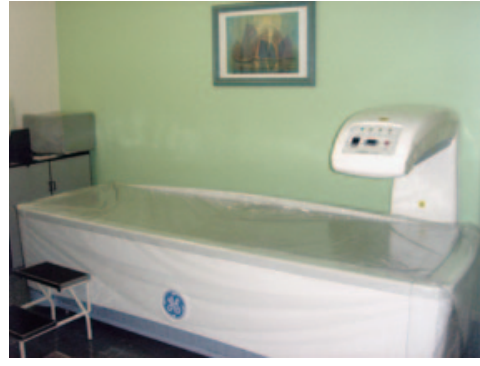

FIGURA 1 - Densitômetro utilizado.

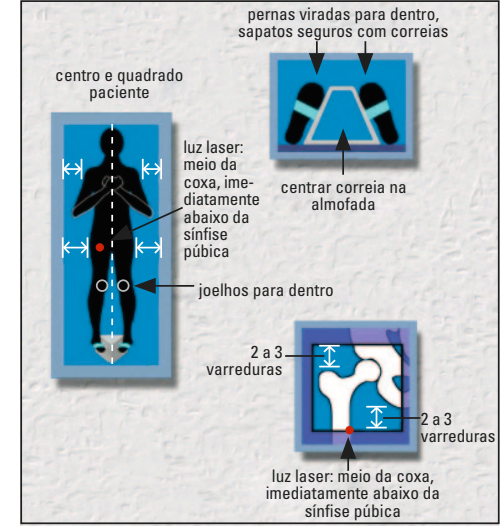

FIGURA 2 - Esquema de posicionamento da paciente para o exame densitométrico do fêmur direito.

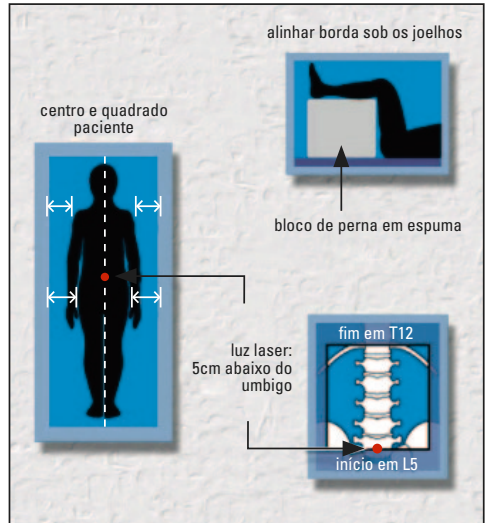

FIGURA 3 - Esquema de posicionamento da paciente para o exame densitométrico da coluna lombar (segmento L1-L4).
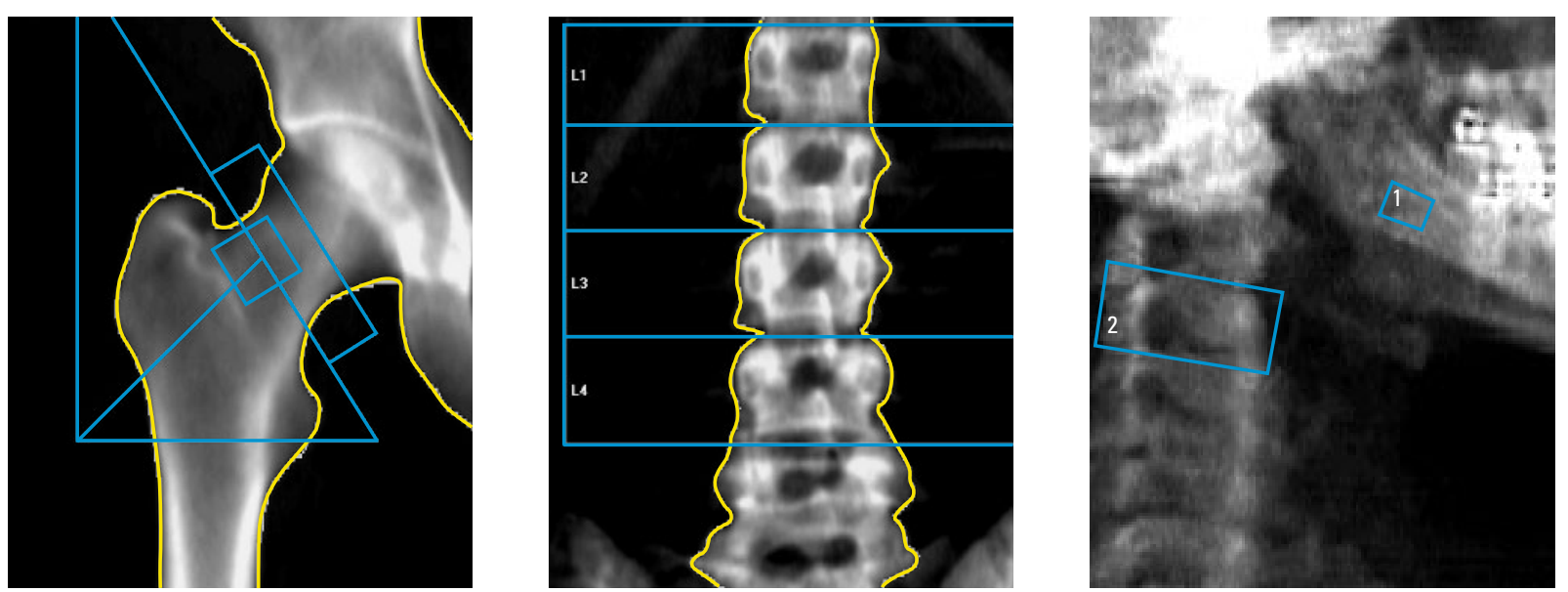

FIGURA 4 - Imagens das ROIs femural, lombar, cervical e mandibular. 


\section{RESULTADOS}

Apresenta-se, na tabela 1, a idade das voluntárias, as leituras de densidades das áreas examinadas e o desvio-padrão em relação ao adulto jovem (índice T) da coluna lombar (segmento L1-L4) e do fêmur total para todos os pacientes da amostra.

A tabela 2 apresenta os índices de correlação entre as quatro áreas avaliadas, ao nível de 5\%.

A tabela 3 demonstra os dados normais obtidos, considerando-se a exclusão dos dois indivíduos que apresentaram mais de um desvio-padrão de discrepância em relação aos valores de referência da Organização Mundial de Saúde para a região do fêmur ou lombar.

TABELA 1 - Idades, valores DMO $\left(\mathrm{g} / \mathrm{cm}^{2}\right)$ dos locais examinados, índices T (lombar e femural) em relação ao adulto jovem, bem como médias e desvios-padrão.

\begin{tabular}{|c|c|c|c|c|c|c|c|c|}
\hline & PACIENTE & IDADE & MANDIBULAR & CERVICAL & LOMBAR & ÍNDICE T (L) & FÊMUR & ÍNDICE T (F) \\
\hline & 1 & 38 & 0,667 & 0,621 & 1,152 & $-0,2$ & 0,913 & $-0,7$ \\
\hline & 2 & 30 & 0,733 & 0,889 & 1,215 & 0,3 & 1,018 & 0,1 \\
\hline & 3 & 43 & 0,953 & 0,526 & 0,918 & $-2,2$ & 0,857 & $-1,2$ \\
\hline & 4 & 38 & 1,410 & 1,086 & 1,130 & $-0,4$ & 1,147 & 1,2 \\
\hline & 5 & 37 & 0,699 & 0,807 & 1,104 & $-0,6$ & 1,033 & 0,3 \\
\hline & 6 & 33 & 1,052 & 0,801 & 1,155 & 0,2 & 0,902 & $-0,8$ \\
\hline & 7 & 30 & 1,399 & 0,830 & 1,202 & 0,2 & 1,007 & 0,1 \\
\hline & 8 & 40 & 1,152 & 0,688 & 1,143 & $-0,3$ & 0,914 & $-0,7$ \\
\hline & 9 & 31 & 1,524 & 0,902 & 1,191 & 0,1 & 1,097 & 0,8 \\
\hline & 10 & 38 & 0,757 & 0,691 & 1,012 & $-1,4$ & 1,084 & 0,7 \\
\hline & 11 & 45 & 0,621 & 0,679 & 1,188 & 0,1 & 0,936 & $-0,5$ \\
\hline & 12 & 44 & 0,547 & 0,743 & 1,072 & $-0,9$ & 0,902 & $-0,8$ \\
\hline & 13 & 45 & 0,681 & 0,726 & 1,198 & 0,1 & 0,922 & $-0,7$ \\
\hline & 14 & 42 & 0,722 & 0,854 & 1,222 & 0,4 & 1,019 & 0,2 \\
\hline & 15 & 36 & 1,522 & 0,871 & 1,237 & 0,5 & 0,943 & $-0,5$ \\
\hline & 16 & 36 & 1,072 & 0,808 & 1,263 & 0,7 & 1,069 & 0,6 \\
\hline & 17 & 38 & 0,758 & 0,735 & 1,311 & 1,1 & 0,936 & $-0,5$ \\
\hline & 18 & 32 & 0,994 & 0,860 & 1,177 & 0,0 & 1,018 & 0,2 \\
\hline & 19 & 41 & 1,103 & 0,797 & 1,302 & 1,0 & 0,895 & $-0,9$ \\
\hline & 20 & 43 & 1,060 & 0,761 & 1,130 & $-0,4$ & 0,882 & $-1,0$ \\
\hline & 21 & 38 & 1,007 & 0,866 & 1,098 & $-0,7$ & 0,885 & $-1,0$ \\
\hline & 22 & 45 & 1,590 & 0,708 & 1,102 & $-0,7$ & 0,905 & $-0,8$ \\
\hline d.p. & $\sigma_{n-1}=$ & 4,912 & 0,323 & 0,116 & 0,091 & 0,764 & 0,082 & 0,688 \\
\hline média & $\bar{X}=$ & 38,3181818 & 1,001 & 0,784 & 1,160 & $-0,141$ & 0,967 & $-0,268$ \\
\hline
\end{tabular}

TABELA 2 - Correlações lineares entre os locais de leitura, ao nível de $5 \%$ de significância.

$\begin{array}{cccc} & \text { MANDIBULAR } & \text { CERVICAL } & \text { LOMBAR } \\ \text { MANDIBULAR } & - & 0,40 & 0,10 \\ \text { CERVICAL } & 0,40 & - & 0,40 \\ \text { LOMBAR } & 0,10 & 0,40 & 0,63^{*} \\ \text { FÊMUR } & 0,18 & 0,63^{*} & 0,14\end{array}$

- estatisticamente significante $(p<0,05)$. 
TABELA 3 - Médias e desvios-padrão dos valores de DMO $\left(\mathrm{g} / \mathrm{cm}^{2}\right)$ mandibular, cervical, lombar e femural das 20 voluntárias com DMO lombar e femural dentro da normalidade.

\begin{tabular}{|c|c|c|c|c|c|}
\hline & PACIENTE & MANDIBULAR & CERVICAL & LOMBAR & FÊMUR \\
\hline & 1 & 0,667 & 0,621 & 1,152 & 0,913 \\
\hline & 2 & 0,733 & 0,889 & 1,215 & 1,018 \\
\hline & 4 & 1,410 & 1,086 & 1,130 & 1,147 \\
\hline & 5 & 0,699 & 0,807 & 1,104 & 1,033 \\
\hline & 6 & 1,052 & 0,801 & 1,155 & 0,902 \\
\hline & 7 & 1,399 & 0,830 & 1,202 & 1,007 \\
\hline & 8 & 1,152 & 0,688 & 1,143 & 0,914 \\
\hline & 9 & 1,524 & 0,902 & 1,191 & 1,097 \\
\hline & 11 & 0,621 & 0,679 & 1,188 & 0,936 \\
\hline & 12 & 0,547 & 0,743 & 1,072 & 0,902 \\
\hline & 13 & 0,681 & 0,726 & 1,198 & 0,922 \\
\hline & 14 & 0,722 & 0,854 & 1,222 & 1,019 \\
\hline & 15 & 1,522 & 0,871 & 1,237 & 0,943 \\
\hline & 16 & 1,072 & 0,808 & 1,263 & 1,069 \\
\hline & 17 & 0,758 & 0,735 & 1,311 & 0,936 \\
\hline & 18 & 0,994 & 0,860 & 1,177 & 1,018 \\
\hline & 19 & 1,103 & 0,797 & 1,302 & 0,895 \\
\hline & 20 & 1,060 & 0,761 & 1,130 & 0,882 \\
\hline & 21 & 1,007 & 0,866 & 1,098 & 0,885 \\
\hline & 22 & 1,590 & 0,708 & 1,102 & 0,905 \\
\hline d.p. & $\sigma_{n-1}=$ & 0,334 & 0,102 & 0,067 & 0,078 \\
\hline média & $\bar{X}=$ & 0,983 & 0,768 & 1,127 & 0,925 \\
\hline
\end{tabular}

\section{DISCUSSÃO}

A literatura é controversa quanto à variabilidade das leituras de densidade óssea nos diferentes sítios esqueléticos ${ }^{11,22,25}$. No presente estudo, os valores de densidade óssea somente se correlacionaram para a região femural e cervical (Tab. 2).

Quanto aos valores de referência da análise realizada no densitômetro da marca Lunar, modelo Prodigy, os critérios de avaliação pela densitometria óssea em fêmur total e em coluna lombar (segmento L1-L4) são obtidos a partir do nível absoluto da densidade mineral óssea (DMO). Esse valor é comparado à DMO do adulto jovem (indivíduos de 25 a 35 anos, do mesmo gênero, peso e etnia do paciente examinado), fornecendo informações sobre o risco de fratura. Os critérios para classificação dos achados densitométricos são baseados na orientação da Organização Mundial de Saúde:

- Índice $\mathrm{T}$ de 0 a $-1,0$ d.p. $\quad=$ normal

- Índice $\mathrm{T}$ de $<-1,0$ a -2,5 d.p. = osteopenia

- Índice $\mathrm{T}$ de $<-2,5$ d.p. $\quad=$ osteoporose

Como esperado, em se tratando de mulheres saudáveis não-menopáusicas, a maioria das pacientes (20) obteve densitometria óssea normal no segmento Ll-L4 da coluna lombar e em fêmur total, com risco de fratura inalterado em relação ao adulto jovem, baseando-se nos valores de referência acima citados. Os gráficos 1 e 2 correspondem, respectivamente, aos laudos dos exames de densitometria do segmento L1-L4 e do fêmur total da paciente \#7, que servem como exemplos 
de valores densitométricos compatíveis com as referências do densitômetro utilizado na pesquisa.

Duas das pacientes examinadas apresentaram DMO reduzida nos segmentos avaliados, o que foi uma surpresa, haja vista que nenhuma paciente encontrava-se no período pós-menopausa. A paciente \#3 apresentou osteopenia no segmento L1L4 de coluna lombar (índice $\mathrm{T}=-2,2$ ) e risco de fratura 4,6 vezes aumentado em relação ao adulto jovem (Gráf. 3), além de uma baixa densidade mineral óssea em fêmur total (índice $\mathrm{T}=-1,2$ ) com risco de fratura 2,3 vezes aumentado em relação ao adulto jovem (Gráf. 4). Esse resultado pode ter sido ocasionado pelo fato da paciente ter amamentado há 2 anos, fato não relatado inicialmente (antes do exame). Porém, não se pode descartar que essa paciente poderia apresentar baixa massa óssea por fatores genéticos, não tendo alcançado um pico de massa óssea adequado para a idade, independentemente do histórico de maternidade e amamentação.

O outro caso foi o da paciente \#10, que, embora tenha apresentado valor densitométrico normal em fêmur total (índice $\mathrm{T}=0,7$ ), apresentou osteopenia no segmento L1-L4 da coluna lombar (índice $\mathrm{T}=-1,4$ ) e risco de fratura 2,6 vezes aumentado em relação ao adulto jovem. Os gráficos referentes aos resultados densitométricos dessa

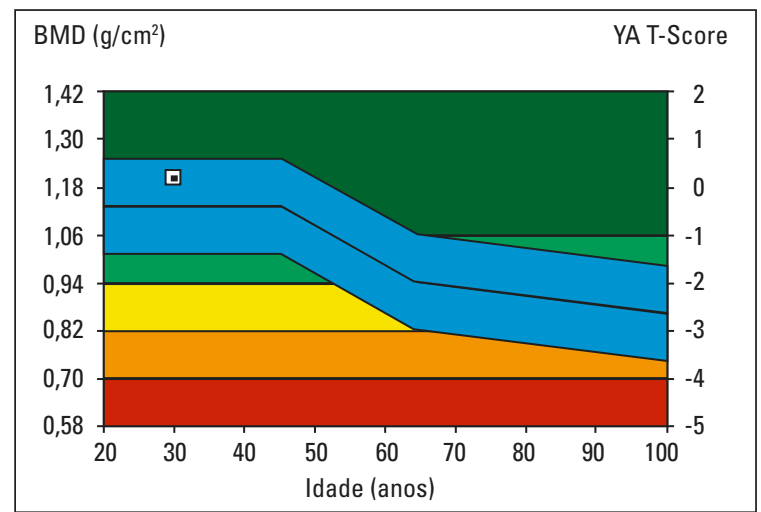

GRÁFICO 1 - Paciente \#7 (L1-L4).

$\operatorname{DM0}\left(\mathrm{g} / \mathrm{cm}^{2}\right)=1,202 ;$ - -score $=0,2 ;$ Z-score $=0,6$. paciente podem ser visualizados nos gráficos 5 e 6 .

Para interpretação desses gráficos, utiliza-se os mesmos valores de referência acima citados, além do desvio-padrão em relação à idade da paciente (índice Z). A faixa azul claro representa os valores densitométricos dentro da normalidade conforme o gênero, idade, peso e etnia da paciente examinada. É importante ressaltar que, no período da menopausa, esses valores tendem a diminuir consideravelmente. As cores verde escuro, verde claro, amarelo, laranja e vermelho das faixas horizontais dos gráficos representam, nessa ordem, a diminuição da densidade óssea, assim como o aumento de risco à fratura das áreas examinadas. Ainda:

- T-score = desvio-padrão em relação ao adulto jovem (Brasil, Coluna AP / Fêmur população de referência, idades 20-40);

- Z-score = desvio-padrão em relação à idade da paciente (correspondência em idade, peso de mulheres entre $25-100 \mathrm{~kg}$ e etnia).

Os valores médios encontrados no presente estudo foram: mandibular $=1,001 \mathrm{~g} / \mathrm{cm}^{2}$; cervical $=0,784 \mathrm{~g} / \mathrm{cm}^{2} ;$ lombar $=1,160 \mathrm{~g} / \mathrm{cm}^{2}$ e femural $=$ $0,967 \mathrm{~g} / \mathrm{cm}^{2}$. Desconsiderando-se os dois casos de alteração da DMO, os dados de referência são respectivamente $0,983 \mathrm{~g} / \mathrm{cm}^{2}$ (d.p. $\left.=0,334\right), 0,768$ g/ $\mathrm{cm}^{2}$ (d.p. = 0,102), 1,127g/ $\mathrm{cm}^{2}$ (d.p. = 0,067), $0,925 \mathrm{~g} / \mathrm{cm}^{2}$ (d.p. $=0,078$ ).

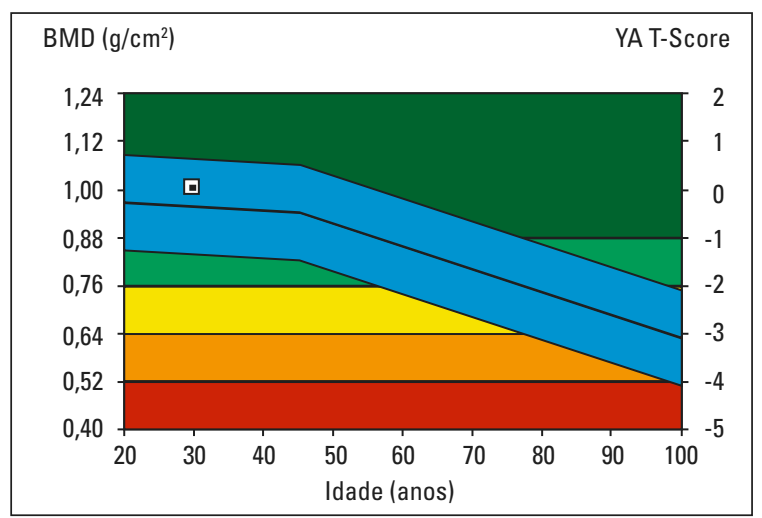

GRÁFICO 2 - Paciente \#7 (Fêmur Total).

DMO $\left(\mathrm{g} / \mathrm{cm}^{2}\right)=1,007 ;$ T-score $=0,1 ;$ Z-score $=0,4$. 


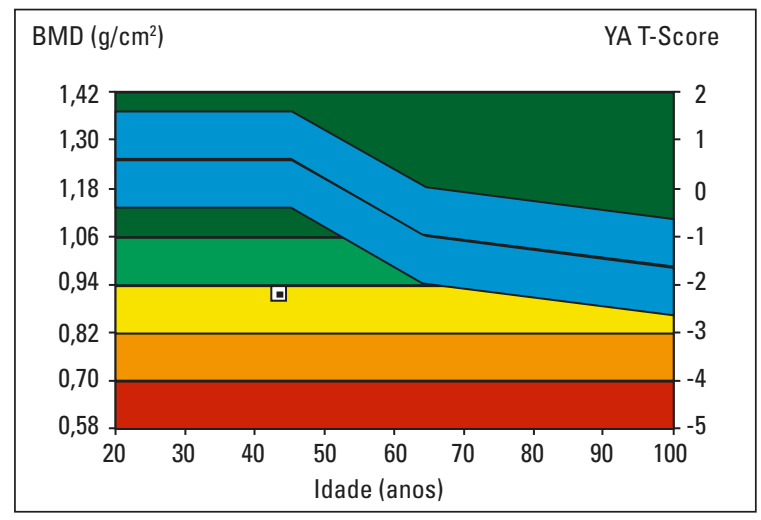

GRÁFICO 3 - Paciente \#3 (L1-L4).

DMO $\left(\mathrm{g} / \mathrm{cm}^{2}\right)=0,918 ;$-score $=-2,2 ;$ Z-score $=-2,7$.

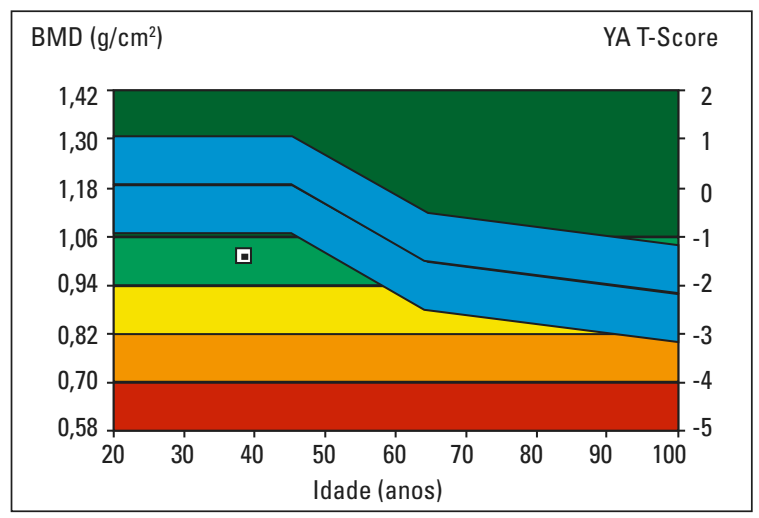

GRÁFICO 5 - Paciente \# 10 (L1-L4).

DMO $\left(\mathrm{g} / \mathrm{cm}^{2}\right)=1,012 ;$ - -score $=-1,4 ;$ Z-score $=-1,4$.

Wahner e Fogelman ${ }^{27}$ relataram que o esqueleto ósseo consiste de 70 a $80 \%$ dos ossos corticais e 20 a 30\% de ossos trabeculados. Essa proporção varia de acordo com a região do esqueleto. Esse fato justifica as variações entre as leituras observadas, bem como as variações individuais, incluindo diferentes idades. Soikkonen, Ainamo e Xie ${ }^{24}$ relataram que fatores múltiplos - como as alterações metabólicas, o padrão nutricional, o status hormonal, o fator genético e as ações mecânicas sobre o rebordo alveolar - influenciam na predisponência da reabsorção óssea em associação ao fator tempo. E Hildebolt ${ }^{8}$ relatou que, embora pareça que

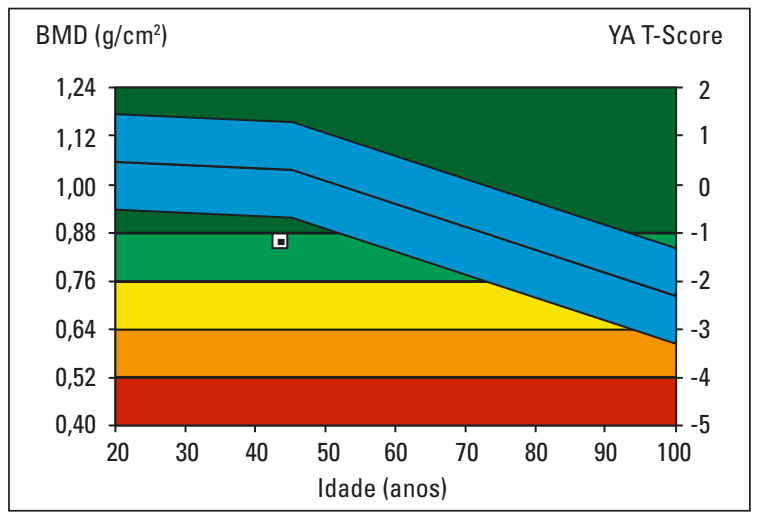

GRÁFICO 4 - Paciente \#3 (Fêmur Total). DMO $\left(\mathrm{g} / \mathrm{cm}^{2}\right)=0,857 ;$ T-score $=-1,2 ;$ - -score $=-1,5$.

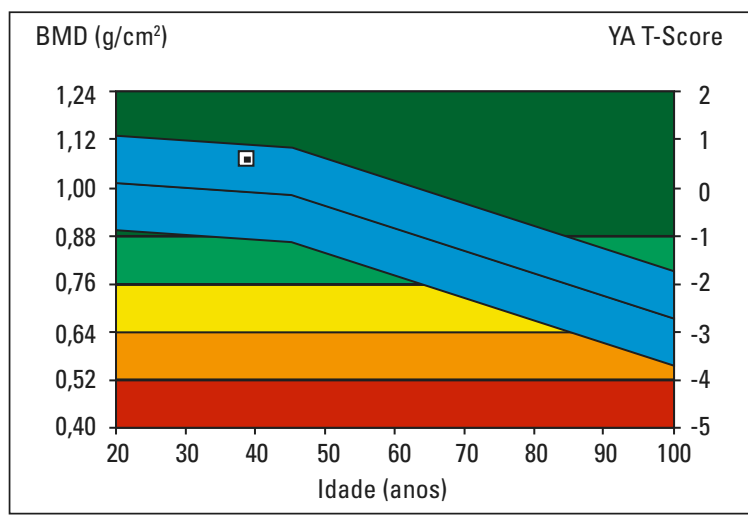

GRÁFICO 6 - Paciente \#10 (Fêmur Total). DMO $\left(\mathrm{g} / \mathrm{cm}^{2}\right)=1,084 ;$ T-score $=0,7 ;$ Z-score $=0,7$.

ocorra uma possível associação entre a osteoporose e a perda alveolar radiográfica, não há evidência clara da correlação de perda de osso alveolar e osteoporose e, para tal confirmação, estudos longitudinais seriam necessários.

Kribbs et al. ${ }^{16}$ compararam os valores da massa óssea, da densidade mandibular, assim como da espessura da cortical óssea na região do ângulo mandibular - em pacientes não-osteoporóticos do gênero feminino pré e pós-menospáusicas - com os valores de massa óssea das vértebras L2 e L4 e do rádio. Concluíram que a massa óssea mandibular era significativamente correlacionada com a massa 
óssea esquelética e relataram que, com o passar da idade, há diminuição da espessura da cortical mandibular. O presente estudo não sustentou estes dados, já que não foi encontrada correlação significativa entre áreas do esqueleto e a região mandibular; além disso, a mostra foi composta apenas de mulheres no período pré-menopausa.

No presente estudo, a delimitação da região de interesse (ROI) mandibular-alveolar não foi capaz de incluir uma região restrita ao processo alveolar, haja vista as sobreposições de estruturas, ainda que a tomada oblíqua tenha sido realizada. Esse fato parece impedir que sejam extrapoladas as leituras mandibulares de modo preciso para a área dentoalveolar de interesse, já que a variação densitométrica pode ocorrer. Entretanto, Verhoeven et $a 1 .{ }^{26}$ observaram que, in vitro, os resultados da densitometria utilizando películas periapicais e radiografias cefalométricas oblíquas (OLCRs) mostraram-se similares. Mas apontaram erros de medida da densitometria com as OLCRs in vivo. Mesmo diante das variações, os autores concluíram que a técnica radiográfica e a da análise das imagens eram ferramentas promissoras para estudos densitométricos, especialmente nas mandíbulas com enxertos ósseos e implantes.

A tomada oblíqua empregada no presente estudo pode justificar o desvio-padrão aumentado para a região mandibular, bem como os diferentes padrões faciais das voluntárias.

Utilizando o método da tomografia computadorizada quantitativa e a densidade mineral óssea da crista alveolar, Lindh, Obrant e Petersson ${ }^{17}$ investigaram correlações entre a DMO de várias regiões da maxila antes do tratamento implantodentário e os valores da coluna e do quadril. Observaram que a $\mathrm{DMO}$ variou significativamente entre os indivíduos $(\mathrm{p}<0,001)$ e nos mesmos indivíduos $(\mathrm{p}<0,001)$. A DMO da região anterior da maxila teve correlação significativa com a DMO da coluna lombar $(r=0,6 ; p<0,05)$. Os autores discutiram que a densidade óssea de um sítio não necessariamente reflete a densidade óssea de outro sítio.
Também demonstraram que, embora a densidade óssea da maxila e a densidade óssea da coluna e do quadril possam ser semelhantes, mais estudos são necessários para investigar essa relação. $\mathrm{O}$ presente estudo aponta para a mesma direção.

Yang et al. ${ }^{29}$ descreveram que a densidade óssea alveolar normal é de $1.850\left(\mathrm{~g} / \mathrm{cm}^{3}\right)$, quando medida diretamente por um dispositivo simplificado associado ao filme periapical. Discutiram que, embora a absorciometria duo-energética por raios X (DXA) seja conhecida por alguns clínicos como padrão-ouro para análise de densidade óssea, seria de difícil acesso para os pacientes odontológicos. Hildebolt et al. ${ }^{9}$ relataram algumas limitações para os exames periapicais, salientando que a avaliação digital de 12 bits na escala de cinza e $50 \mu \mathrm{m}$ de resolução é recomendada para a densitometria de osso alveolar realizada com radiografias Ektaspeed. No presente estudo, em que foi utilizado um densitômetro DXA, observou-se que se trata de um exame simples e que, em virtude da demanda aumentada, bem como da facilidade de acesso por planos de saúde, pode ser também acessível para os pacientes. Entretanto, sugere-se que o padrão de exame inclua, além das áreas tradicionais femural e lombar, também a área mandibular. Observa-se, ainda, que o valor obtido por Yang et al. ${ }^{29}$ foi de $1.850 \mathrm{~g} / \mathrm{cm}^{3}$, praticamente o dobro do observado pelo presente estudo. Embora tenham calculado uma unidade cúbica em um exame bidimensional, mais trabalhos são necessários para detalhar essa diferença.

Garcia e Souza ${ }^{6}$ relacionaram os valores lineares da reabsorção da crista óssea alveolar e os valores lineares da espessura cortical vestibular para o osso mandibular em radiografias digitais indiretas com valores de densidade óssea da coluna lombar (L2-L4) e fêmur total proximal direto. Também avaliaram mulheres pré-menopáusicas e mostraram que a reabsorção da crista óssea alveolar, mesmo em indivíduos normais, está mais relacionada à idade ( $\mathrm{p}=0,001)$, e que não foi observada significância $(p=0,05)$ para a correlação dos valores de 
espessura da cortical óssea e densitometria, corroborando o presente estudo.

Os formatos das raízes dentárias e da crista alveolar foram mencionados por Consolaro ${ }^{3}$ como fatores que podem influenciar na resposta biológica diante da movimentação ortodôntica. Esses fatores estariam relacionados com o grau de concentração de forças nas regiões apicais radiculares. Considerou que as raízes mais triangulares e cristas alveolares mais retangulares acarretariam uma maior concentração de força no ápice radicular, favorecendo o processo de reabsorção do cemento. Horiuchi, Hotokezaka e Kobayashi ${ }^{10}$ ressaltaram, também, que a movimentação dentária contra uma área de maior densidade óssea - por exemplo, a cortical óssea pode estar relacionada à maior reabsorção dentária.

Criando-se um índice $\mathrm{T}$ para os valores DMO aqui encontrados (Gráf. 7), e considerando-se o desvio-padrão próximo de $0,3 \mathrm{~g} / \mathrm{cm}^{2}$, seria razoável supor que, se o valor mandibular apresentar-se acima de $1,3 \mathrm{~g} / \mathrm{cm}^{2}$, a densidade estaria aumentada e, ao contrário, se apresentar-se abaixo de $0,7 \mathrm{~g} / \mathrm{cm}^{2}$, poder-se-ia considerar uma densidade baixa.

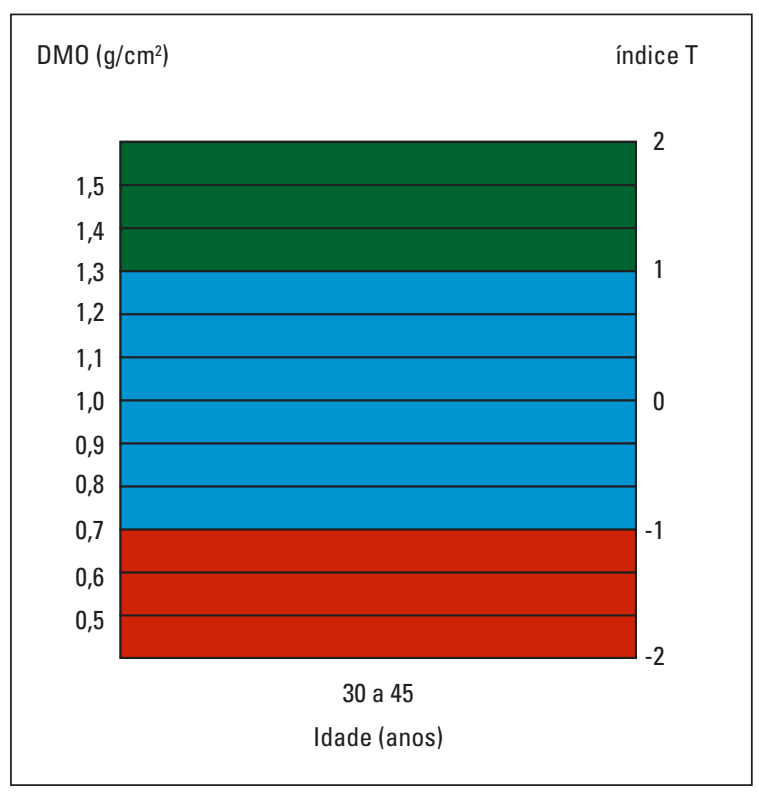

GRÁFICO 7 - Gráfico ilustrando a variação densitométrica mandibular encontrada para a normalidade e os seus limites baseados no desviopadrão para a amostra estudada.
Considerando esses valores, os pacientes de número 4, 7, 9, 15 e 22 apresentam-se com densidade aumentada e os pacientes de número 1, 5, 11, $12 \mathrm{e}$ 13 apresentam-se com densidade diminuída

Diante da possibilidade de que as diferenças nas densidades alveolares mandibulares e maxilares possam variar e interferir, de alguma forma, no prognóstico do ritmo e repercussão da movimentação ortodôntica, sugere-se que o exame densitométrico mandibular possa ser um instrumento de ampliação prognóstica. Para tanto, mais estudos contribuirão para a aplicabilidade desse exame.

\section{CONCLUSÃO}

Não houve correlação significativa entre a densidade mandibular e as demais áreas estudadas. Houve correlação significativa apenas entre a região cervical e a femural.

O valor médio DMO normal para a região mandibular foi de $0,983 \mathrm{~g} / \mathrm{cm}^{2}$ (d.p. $=0,334$ ), enquanto para a região cervical foi de $0,768 \mathrm{~g} / \mathrm{cm}^{2}$ (d.p. $=0,102)$, e os valores médios para a região lombar e femural foram de, respectivamente, $1,127 \mathrm{~g} / \mathrm{cm}^{2}$ (d.p. $\left.=0,067\right), 0,925 \mathrm{~g} / \mathrm{cm}^{2}$ (d.p. $=$ 0,078 ), esses últimos semelhantes aos valores de referência da OMS.

O exame da área femural pode abranger o valor esperado para a área cervical, entretanto há necessidade do exame densitométrico particular para a área mandibular.

Estudos adicionais são necessários para avaliar as variações locais e a eventual influência sobre a movimentação ortodôntica.

\section{AGRADECIMENTOS}

Os autores agradecem à técnica Claudia de Fátima Nicoletti e à estatística Valentina de Lourdes Milani de Paula Soares. 


\title{
Correlation among mandibular, femoral, lumbar and cervical bone density
}

\begin{abstract}
Introduction: Due to the rise in frequency of adult patients who currently are submitted to orthodontic treatment, general health conditions of this age have been a reason of inquiries correlated to events related to bone metabolism, as dental movements are dependent on the process of bone remodeling, even though in a local level. Different standards of bone density can give different answers to the orthodontic movement. Aim: The present study evaluated the correlation of the general bone mineral density (BMD) to the mandibular region. Methods: Therefore, 22 healthy women aged between 30 and 45 years old were selected for bone densitometry examinations of lumbar, cervical, femoral, as well as mandibular alveolar region. The correlations to these readings were tested as well as values of reference were established for cervical and mandibular areas. Results: The results did not demonstrate significant correlation among the mandibular density to the others studied areas. There was only significant correlation between cervical and femural region. Normal BMD average value for mandibular region was $0.983 \mathrm{~g} / \mathrm{cm}^{2}(S D=0.334)$, whereas for cervical region was $0.768 \mathrm{~g} / \mathrm{cm}^{2}(S D=0.102)$, and the average values for lumbar and femoral regions were respectively $1.127 \mathrm{~g} / \mathrm{cm}^{2}(S D=0.067)$ and $0.925 \mathrm{~g} / \mathrm{cm}^{2}(S D=0.078)$, these last ones were similar to the reference values of World Health Organization (WHO). Conclusions: It is suggested that the examination of the femoral area can comprehend the expected value to cervical area, however particular densitometry examination for the mandibular area is needed, and the exploration of the traditional values (lumbar and femoral) is not appropriate to the estimative of this area. Additional studies are necessary to evaluate local density variations and its influence on orthodontic movement.
\end{abstract}

Keywords: Bone densitometry. Osteoporosis. Orthodontic movement. Bone remodeling. Mandibular bone density.

\section{REFERÊNCIAS}

1. APRIL, H.; FIGÚN, M.; GARINO, R. Anatomia odontológica orocervicofacial. 5. ed. Buenos Aires: El Ateneu, 1975.

2. ATKINSON, P. J.; WOODHEAD, C. Changes in human mandibular structure with age. Arch. Oral Biol., Oxford, v. 13, p. $1453-1463,1968$.

3. CONSOLARO, A. Reabsorções dentárias nas especialidades clínicas. 2. ed. Maringá: Dental Press, 2005.

4. DOVE, S. B. Digital imaging in Dentistry. Dental Diagnostic Science. San Antonio: Uthscsa, 1995. Disponível em: <http://www. ddsx.uthsesa.edu/dig/digtutor.htm>. Acesso em: 22 jan. 2009.

5. GARCIA, R. S. Densidade óssea: estudo da área entre incisivos laterais e caninos na maxila em humanos. 1995. 126 f. Dissertação (Mestrado em Cirurgia e Traumatologia Bucomaxilofacial)Pontifícia Universidade Católica do Rio Grande do Sul, Porto Alegre, 1995.

6. GARCIA, R. S.; SOUZA, A. C. A. Análise quantitativa de sítios mandibulares e sua correlação com valores densitométricos e ultra-sonométricos de estruturas ósseas axiais e periféricas em mulheres pré-menopáusicas. Rev. Odonto Cienc., Porto Alegre, v. 14, n. 27, p. 289-327, jun. 1999.
7. HAUSMANN, E. A contemporary perspective on techniques for the clinical assessment of alveolar bone. J. Periodontol., Chicago, v. 61, no. 3, p. 149-156, Mar. 1990.

8. HILDEBOLT, C. F. Osteoporosis and oral bone loss. Dentomaxillofac. Radiol., Tokyo, v. 26, no. 1, p. 3-15, Jan. 1997.

9. HILDEBOLT, C. F.; BARTLETT, T. Q.; BRUNSDEN, B. S; HENTE, N. L.; GRAVIER, M. J.; WALKUP, R. K.; SHROUT, M. K.; VANNIER, M. W. Bitewing-based alveolar bone densitometry: digital imaging resolution requirements. Dentomaxillofac. Radiol., Tokyo, v. 23, no. 3, p. 129-134, Aug. 1994

10. HORIUCHI, A.; HOTOKEZAKA, H.; KOBAYASHI, K. Correlation between cortical plate proximity and apical root resorption. Am. J. Orthod. Dentofacial Orthop., St. Louis, v. 114, no. 3, p. 311-318, Sept. 1998.

11. HORNER, K. et al. Mandibular bone mineral density as a predictor of skeletal osteoporosis. Br. J. Radiol., London, v. 69, no. 827, p. 1019-1025, Nov. 1996.

12. ISENBARGER, D.; CHAPIN, B. Osteoporosis: Current pharmacologic options for prevention and treatment. Postgrad. Med., Berwyn, v. 101, no. 1, p. 129-142, Jan. 1997. 
13. KANIS, J. Osteoporosis. Cambridge: Blackwell Science, 1996.

14. KLEEREKOPER, M. The epidemiology of osteoporosis. In: ROSEN, C. Osteoporosis: diagnostics and therapeutic principles. New Jersey: Human Press, 1996. p. 65

15. KRIBBS, P. J. et al. Comparison of mandibular bone in normal and osteoporotic women. J. Prosthet. Dent., St. Louis, v. 63, no. 2, p. 218-222, Feb. 1990

16. KRIBBS, P. J. et al. Relationships between mandibular and skeletal bone in a population of normal women. J. Prosthet. Dent., St. Louis, v. 32, no. 1, p. 105, June 1990.

17. LINDH, C.; OBRANT, K.; PETERSSON, A. Maxillary bone mineral density and its relationship to the bone mineral density of the lumbar spine and hip. Oral Surg. Oral Med. Oral Pathol. Oral Radiol. Endod., St. Louis, v. 98, no. 1, p. 102-109, July 2004.

18. LIPS, P. Epidemiology and predictors of fractures associated with osteoporosis. Am. J. Med., New York, v. 103, no. 2A p. 35-115, Aug. 1997.

19. MOUYEN, F. et al. Presentation and physical evaluation of radiovisiography. Oral Surg. Oral Med. Oral Pathol., St. Louis, v. 68, no. 2, p. 238-242, 1989

20. NATIONAL OSTEOPOROSIS FOUNDATION. Osteoporosis: A debilitating disease that can be prevented and treated. Disponivel em: <http://www.nof.org/osteoporosis/index.htm>. Acesso em: 22 jan. 2009

21. PADILHA, D. M. P. A saúde bucal de pacientes idosos: aspectos clínicos de um grupo de idosos ingleses e clínico-radiográfico de um grupo de idosos brasileiros. 1997. $260 \mathrm{f}$. Tese (Doutorado em Estomatologia Clínica)-Pontifícia Universidade Católica do Rio Grande do Sul, Porto Alegre, 1997.

22. POLIDO, W. D. Avaliação das alterações ósseas ao redor de implantes dentários durante o período de osseointegração através da radiografia digital direta. 1997. $174 \mathrm{f}$. Tese (Doutorado em Cirurgia e Traumatologia Bucomaxilofacial)-Pontifícia Universidade Católica do Rio Grande do Sul, Porto Alegre, 1997.
23. SCARPARO, H. C. Apresentação de uma metodologia exploratória para análise de densidade óssea na mandíbula. 1995. 101 f. Dissertação (Mestrado em Cirurgia e Traumatologia Bucomaxilofacial)-Pontifícia Universidade Católica do Rio Grande do Sul, Porto Alegre, 1995

24. SOIKKONEN, K.; AINAMO, A.; XIE, Q. Height of residual ridge and radiographic structure in jaws of clinically edentulous elderly people. J. Oral Rehabil., Oxford, v. 23, no. 7, p. 470-475, 1996

25. SOUZA, A. C. A. Diagnóstico da osteoporose. In: SIMPÓSIO DE ATUALIZAÇÃO DE OSTEOPOROSE. Novo Hamburgo, 1996. p. 7-9

26. VERHOEVEN, J. W.; RUIJTER, J.; CUNE, M. S.; TERLOU, M. Oblique lateral cephalometric radiographs of the mandible in Implantology: usefulness and reproducibility of the technique in quantitative densitometric measurements of the mandible in vivo. Clin. Oral Implants Res., Copenhagen, v. 11, no. 5, p. 476-486, Oct. 2000

27. WAHNER, H. W.; FOGELMAN, I. The evaluation of osteoporosis: dual energy $x$-ray absorptiometry in clinical practice. London: Martin Dunitz, 1994.

28. WAHNER, H. W.; STEIGER, P.; VON STETTEN, E.; WAHNER, H. W.; FOGELMAN, I. The evaluation of osteoporosis: dual energy $\mathrm{x}$-ray absorptiometry in clinical practice. 1994. chap. 3, p. 14-34

29. YANG, J.; CHIOU, R.; RUPRECHT, A.; VICARIO, J.; MAcPHAIL, L. A.; RAMS, T. E. A new device for measuring density of jaw bones. Dentomaxillofac Radiol., Houndsmills, v. 31, no. 5 , p. 313-316, Sept. 2002.
Endereço para correspondência

Paula Cabrini Scheibe

Rua Mem de Sá - 511 - Zona 2

CEP: 87.010 - 3700 - Maringá / PR

E-mail: pscheibel@ortodontista.com.br 